\title{
Combination of Baicalein and Amphotericin B Accelerates Candida albicans Apoptosis
}

\author{
ZiJin Fu, Hui Lu, ZhenYu Zhu, Lan Yan, YuanYing JiAnG, and YingYing CAO* \\ School of Pharmacy, Second Military Medical University; 325 Guohe Road, Shanghai, 200433, P. R. China.
}

Received October 8, 2010; accepted November 24, 2010; published online November 26, 2010

\begin{abstract}
Candida albicans is the most important human fungal pathogen. Amphotericin (AmB) is a gold standard of antifungal treatment for fungi, but the severe side effect of this drug restricts its clinical application. In this study, the interaction of $\mathrm{AmB}$ and baicalein (BE) was investigated against thirty clinical isolates of $C$. albicans. Synergistic activities were determined using the checkerboard microdilution assay based on the fractional inhibitory concentration indices. Combination of $\mathrm{BE}$ and $\mathrm{AmB}$ accelerated $C$. albicans apoptosis accompanied with an increase of reactive oxygen species (ROS). Moreover, $\mathrm{AmB}$ increased the caspase activity and expression of the corresponding gene $\mathrm{CaMCA1}$ in $\mathrm{C}$. albicans. These effects were enhanced in the presence of BE. Deletion of CaMCA1 clearly attenuated AmB-induced apoptosis, indicating the involvement of $C a M C A 1$-mediated caspase pathway in AmB-induced apoptosis and the synergistic action.
\end{abstract}

Key words Candida albicans; amphotericin; baicalein; synergism; caspase

Candida albicans is the most important human fungal pathogen, causing various diseases from superficial mucosal infections to life-threatening systemic disorders. ${ }^{1,2)}$ Compared to bacterial infections, few drugs are available to treat fungal infections. The polyene macrolide antibiotic amphotericin $\mathrm{B}(\mathrm{AmB})$ is effective in the treatment of many systemic mycoses. But due to its poor permeability across the membrane, an increased amount of AmB must be administered to patients in clinical situations, thus often resulting in severe side effects. ${ }^{3-5}$ ) To lessen the severity of the side effects, AmB is often combined with other antifungal drugs such as the azoles, but coincident with the increased use of antifungal azole derivatives, the incidences of drug resistance have been recently increasing. ${ }^{6-8)}$ Thus investigation of reducing the AmB dose by combining it with other compounds is necessary.

Programmed cell death responses have been described in a variety of fungi after exposure of the organisms to a range of conditions such as weak acid stress, oxidative stress, or ultraviolet irradiation. $^{9-11)}$ It was demonstrated that $C$. albicans could be triggered to undergo an apoptotic cell death response when exposed to $\mathrm{H}_{2} \mathrm{O}_{2}$, intracellular acidification, or AmB. A single-cell death assay was used to show that a majority of AmB-treated cells were nonviable and impermeable to propidium iodide. Apoptotic changes were further confirmed by electron microscopy and by the production of intracellular reactive oxygen species (ROS). ${ }^{12,13)}$ However, the mechanism by which $C$. albicans undergoes apoptosis remains unclear. In mammalian cells, acquisition of an apoptotic morphology depends on the activation of cysteine dependent aspartate specific proteases (caspases). Functional analyses of genes in Saccharomyces cerevisiae have revealed similarities between fungal apoptosis and apoptosis in mammalian cells. A homologue of mammalian caspases, YCA1 (Yeast Caspase 1), has been identified. Overexpression of YCA1 increases hydrogen peroxide-induced caspase-like activity and apoptosis in $S$. cerevisiae. ${ }^{14-17)}$ Caspase superfamily consists of three families; YCA1 belongs to the metacaspase family of enzymes which are found in plants, fungi and protozoa. We recently found that CaMCA1, a homologue of
S. cerevisiae metacaspase $Y C A 1$, was involved in oxidative stress-induced apoptosis in C. albicans. ${ }^{18)}$

Baicalein (BE) is a compound that was originally extracted from the Scutellaria baicalensis root, and it is a promising herbal medicine in Chinese Pharmacopoeia (Fig. 1). BE exhibits antioxidant, antibacterial, antiviral, and antifungal activities. ${ }^{19-23)}$ In this study, we described a synergic effect of $\mathrm{BE}$ with $\mathrm{AmB}$ against $C$. albicans and the potential mechanism. Our results showed that combination of $\mathrm{BE}$ and $\mathrm{AmB}$ enhanced $C$. albicans apootosis accompanied with an increase of reactive oxygen species (ROS). We also found that AmB could induce the caspase activity and mRNA expression of the gene encoding $C$. albicans caspase, CaMCA1. These effects were markedly enhanced when BE was added. Furthermore, we found that deletion of CaMCA1 clearly attenuated AmB-induced caspase activity and apoptosis regardless of the presence or absence of $\mathrm{BE}$, indicating the involvement of CaMCA1-mediated caspase pathway in AmB-induced apoptosis and the synergistic action.

\section{MATERIALS AND METHODS}

Strains and Agents Thirty clinical isolates of fluconazole-resistant $C$. albicans were used in this study, including previously published isolates $\mathrm{FH} 1$ and TL1. ${ }^{24-26)} \mathrm{BE}$ (Sigma-Aldrich, St. Louis, MO, U.S.A.) and AmB (SigmaAldrich, St. Louis, MO, U.S.A.) were dissolved in dimethyl sulfoxide. The strains were cultivated at $30^{\circ} \mathrm{C}$ under constant shaking $(200 \mathrm{rpm})$ in a liquid complete medium YPD consisting of $1 \%(\mathrm{w} / \mathrm{v})$ yeast extract, $2 \%(\mathrm{w} / \mathrm{v})$ peptone, and $2 \%$ (w/v) dextrose.

Checkerboard Microdilution Assay Assays were performed on all 30 isolates according to methods of the CLSI

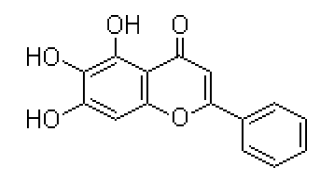

Fig. 1. Structure of Baicalein (BE) 
(formerly NCCLS) (M27-A2). ${ }^{27)}$ The initial concentration of fungal suspension in RPMI 1640 medium was $10^{3} \mathrm{CFU} / \mathrm{ml}$, and the final concentrations ranged from 0.0029 to $1.5 \mu \mathrm{g} / \mathrm{ml}$ for $\mathrm{AmB}$ and 0.0625 to $32 \mu \mathrm{g} / \mathrm{ml}$ for BE. Plates were incubated at $35^{\circ} \mathrm{C}$ for $24 \mathrm{~h}$. Optical density was measured at $630 \mathrm{~nm}$, and background optical densities were subtracted from that of each well. Each isolate was tested in triplicate. Minimum inhibitory concentrations $\left(\mathrm{MIC}_{80}\right.$ and $\mathrm{MIC}_{50}$ ) were determined as the lowest concentration of the drugs (alone or in combination) that inhibited growth by $80 \%$ and $50 \%$ respectively were observed. The fractional inhibitory concentration (FIC) index is defined as the sum of the MIC of each drug when used in combination divided by the MIC of the drug used alone. Synergism and antagonism were defined by FIC indices of $\leq 0.5$ and $>4$, respectively. An FIC index result of $>0.5$ but $\leq 4$ was considered indifferent. ${ }^{28)}$

Cell Death Measurement C. albicans 0610215 was grown in YPD medium with vigorous shaking at $30^{\circ} \mathrm{C}$. Cells grown to early exponential phase were incubated with $\mathrm{AmB}$ $(0.5 \mu \mathrm{g} / \mathrm{ml}), \mathrm{BE}(2 \mu \mathrm{g} / \mathrm{ml})$, combination of $\mathrm{AmB}(0.5 \mu \mathrm{g} / \mathrm{ml})$ and $\mathrm{BE}(2 \mu \mathrm{g} / \mathrm{ml})$ for $4 \mathrm{~h}$ and then harvested for death measurement. Protoplasts of $C$. albicans were stained with propidium iodide (PI) and FITC-labeled annexin-V by using the Annexin-V-FLUOS kit (Roche Applied Science, Germany) to assess necrosis (cellular integrity) and apoptosis (externalization of phosphatidylserine). ${ }^{12)}$ The PI and annexin status of a minimum of 100 protoplasts were recorded for each treatment.

Measurement of ROS Levels Intracellular levels of ROS were measured with DCFH-DA (Molecular Probes, U.S.A.). Briefly, cultured cells were collected by centrifugation and washed with PBS. Subsequently, the cells were adjusted to $2 \times 10^{7}$ cells $/ \mathrm{ml}$. After being incubated with 20 $\mu \mathrm{g} / \mathrm{ml}$ of dichlorofluorescein diacetate (DCFH-DA) for 30 min at $30^{\circ} \mathrm{C}$, the cells were collected by centrifugation and washed with PBS. Then the cells were exposed to the compounds and incubated at $30^{\circ} \mathrm{C}$ with constant shaking (200 rpm). At specified interval, cell samples were observed with a Leica TCS $s p^{2}$ confocal scanning laser microscope with excitation at $485 \mathrm{~nm}$ and emission at $520 \mathrm{~nm}$. On another experiment, cell suspensions were harvested and transferred to the wells of a flat-bottom microplate (BMG Microplate, 96well, Blank) to detect fluoresence intensity on the POLARstar Galaxy (BMG, Labtech, Offenburg, Germany) with excitation at $485 \mathrm{~nm}$ and emission at $520 \mathrm{~nm}$.

Real-Time RT-PCR RNA isolation and real-time RTPCR were performed as described previously. ${ }^{29)}$ Isolated RNA was resuspended in diethyl pyrocarbonate-treated water. The $\mathrm{OD}_{260}$ and $\mathrm{OD}_{280}$ were measured, and the integrity of the RNA was visualized by subjecting 2 to $5 \mu \mathrm{l}$ of the samples to electrophoresis through a $1 \%$ agarose-MOPS gel. First-strand cDNAs were synthesized from $3 \mu \mathrm{g}$ of total RNA in a $60 \mu \mathrm{l}$ reaction volume using the cDNA synthesis kit for RT-PCR (TaKaRa Biotechnology, Dalian, P. R. China) in accordance with the manufacturer's instructions. Triplicate independent quantitative real-time PCR were performed using the LightCycler System (Roche diagnostics, GmbH Mannheim, Germany). SYBR Green I (TaKaRa, Japan) was used to visualize and monitor the amplified product in real time according to the manufacturer's protocol. CaMCA1 was amplified with the forward primer 5'-TATAATAGACCTT-
CTGGAC-3' and the reverse primer 5'-TTGGTGGACGAGAATAATG-3'.

The PCR protocol consisted of denaturation program $\left(95^{\circ} \mathrm{C}\right.$ for $\left.10 \mathrm{~s}\right), 40$ cycles of amplification and quantification program $\left(95^{\circ} \mathrm{C}\right.$ for $10 \mathrm{~s}, 60^{\circ} \mathrm{C}$ for $20 \mathrm{~s}, 72^{\circ} \mathrm{C}$ for $15 \mathrm{~s}$ with a single fluorescence measurement), melting curve program $\left(60-95^{\circ} \mathrm{C}\right.$ with a heating rate of $0.1^{\circ} \mathrm{C} / \mathrm{s}$ and a continuous fluorescence measurement) and finally a cooling step to $40{ }^{\circ} \mathrm{C}$. A standard curve for each primer set was performed with $1: 10,1: 25,1: 50,1: 100,1: 250$ and $1: 500$ dilutions of the cDNA. The slopes of the standard curves were within $10 \%$ of $100 \%$ efficiency. The change in fluorescence of SYBR Green I dye in every cycle was monitored by the LightCycler system software, and the threshold cycle $\left(C_{\mathrm{T}}\right)$ above background for each reaction was calculated. The $C_{\mathrm{T}}$ value of $A C T 1$ (amplified with the forward primer 5'-CAACAAGGACAATACAATAG- $3^{\prime}$ and the reverse primer $5^{\prime}$ GTTGGTGGACGAGAATAATG-3') was subtracted from that of the tested genes to obtain a $\Delta C_{\mathrm{T}}$ value. The $\Delta C_{\mathrm{T}}$ value of an arbitrary calibrator was subtracted from the $\Delta C_{\mathrm{T}}$ value of each sample to obtain a $\Delta \Delta C_{\mathrm{T}}$ value. The gene expression level relative to the calibrator was expressed as $2^{-\Delta \Delta C_{\mathrm{T}}}$.

Assessment of Caspase Activity Caspase activity was detected by staining with $\mathrm{D}_{2} \mathrm{R}$ (CaspSCREEN Flow Cytometric Apoptosis Detection Kit, BioVision, U.S.A.), the nonfluorescent substrate, which is cleaved to green fluorescent monosubstituted rhodamine 110 and free rhodamine. ${ }^{30)}$ Cells were in $\mathrm{D}_{2} \mathrm{R}$ incubation buffer at $30^{\circ} \mathrm{C}$ for $45 \mathrm{~min}$ before viewing and counting under a fluorescence microscope.

Statistical Criteria The MICs data are reported as the median, the range of the concentration of each antifungal agent inhibiting $50 \%\left(\mathrm{MIC}_{50}\right)$ and $80 \%\left(\mathrm{MIC}_{80}\right)$ of the isolates tested. Other data are expressed as mean \pm standard deviation (S.D.). $p<0.01$ was considered to be statistically significant.

\section{RESULTS}

BE Synergy with AmB against $C$. albicans The susceptibilities of $C$. albicans to AmB in the absence or presence of $\mathrm{BE}$ were confirmed by determining the lowest drug concentrations that gave $50 \%\left(\mathrm{MIC}_{50}\right)$ or $80 \%$ inhibition $\left(\mathrm{MIC}_{80}\right)$. The results of the checkerboard analysis are summarized in Table 1. Both AmB and baicalein showed antifungal activity when tested alone. However, the AmB-BE combination markedly reduced the MICs. Synergism was observed in all 30 isolates $(100 \%)$ in terms of $\mathrm{MIC}_{80}$ s. The corresponding median FIC index was 0.053 (range, 0.031 to 0.677). When analyzed with $\mathrm{MIC}_{50} \mathrm{~s}$ instead of $\mathrm{MIC}_{80} \mathrm{~s}$, the FIC index was generally higher (median, 0.171 ; range, 0.033 to 0.965$)$. Of the 30 isolates, synergism was observed in 27 $(90 \%)$, and indifference was observed in $3(10 \%)$. Regardless of MIC endpoints, antagonism was not observed in the combination.

Combination of BE and AmB Enhances $C$. albicans Apoptosis It has been reported that either $\mathrm{BE}$ or $\mathrm{AmB}$ treated alone could induce $C$. albicans apoptosis. ${ }^{12,31)}$ Here we determined this effect of $\mathrm{BE}$ and $\mathrm{AmB}$ treated in combination on one randomly chosen $C$. albicans clinical isolate 0610215. As shown in Fig. 2, after treatment with $2 \mu \mathrm{g} / \mathrm{ml}$ $\mathrm{BE}$ for $4 \mathrm{~h}$, the death rate of the cells was $11 \%$ (7\% as apo- 
Table 1. Interaction of AmB and Baicalein (BE) against 30 Clinical Isolates of C. albicans by Checkerboard Microdilution Assay ${ }^{a}$

\begin{tabular}{|c|c|c|c|c|}
\hline \multirow{2}{*}{ Parameter } & \multicolumn{2}{|c|}{$\mathrm{MIC}_{50}(\mu \mathrm{g} / \mathrm{ml})$} & \multicolumn{2}{|c|}{$\operatorname{MIC}_{80}(\mu \mathrm{g} / \mathrm{ml})$} \\
\hline & Median & Range & Median & Range \\
\hline $\mathrm{AmB}$ & 0.375 & $0.094-0.75$ & 0.75 & $0.188-1.5$ \\
\hline $\mathrm{BE}$ & 2 & $1-8$ & 4 & $2-32$ \\
\hline $\mathrm{AmB}+\mathrm{BE}^{b)}$ & $0.0117 / 0.25$ & $<0.0029-0.0465 / 0.0625-0.25$ & $0.0234 / 0.5$ & $0.0058-0.0938 / 0.125-0.5$ \\
\hline FIC index & 0.171 & $0.033-0.965$ & 0.053 & $0.031-0.677$ \\
\hline
\end{tabular}

a) When analyzed with $\mathrm{MIC}_{50}$, synergism was observed in 27 of 30 isolates ( $90 \%$ ) and indifference was observed in 3 of the 30 isolates (10\%); at $\mathrm{MIC}_{80}$, synergy was observed in all of the isolates. b) MICs in combination are expressed as [AmB]/[BE].

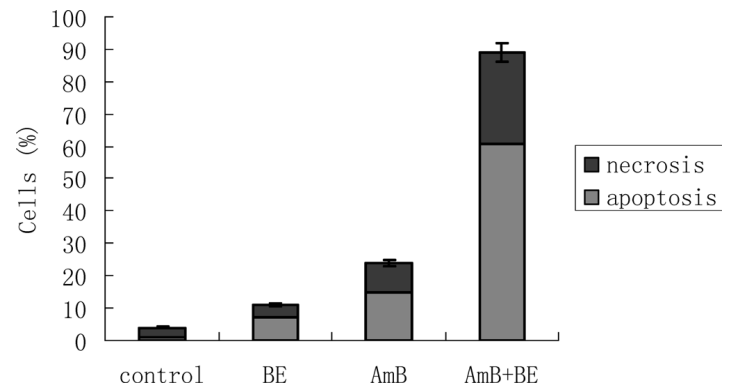

Fig. 2. Effect of AmB and BE on Cell Death

C. albicans 0610215 cells were exposed to $0.5 \mu \mathrm{g} / \mathrm{ml} \mathrm{AmB}, 2 \mu \mathrm{g} / \mathrm{ml} \mathrm{BE}$ or a combination for $4 \mathrm{~h}$. Propidium iodide (PI) and FITC-labeled annexin-V were used to determine the rate of cell death, including apoptotic annexin (+) PI $(-)$, and necrotic annexin $(+)$ PI $(+)$ cells. These data were mean values \pm S.D. from three independent experiments.

ptosis and $4 \%$ as necrosis). Treatment of $0.5 \mu \mathrm{g} / \mathrm{ml} \mathrm{AmB}$ resulted in $24 \%$ of death rate $(15 \%$ as apoptosis and $9 \%$ as necrosis). When $\mathrm{BE}$ and $\mathrm{AmB}$ were used in combination, the death rate was $89 \%(61 \%$ as apoptosis and $28 \%$ as necrosis), indicating a synergy of $\mathrm{BE}$ and $\mathrm{AmB}$ on $C$. albicans apoptosis.

Combination of BE and AmB Enhances ROS Production Since ROS are intently correlated with the cell apoptosis, we investigated ROS generation of the cells with the fluorescent dye DCFH-DA. As shown in Fig. 3, the ROS level in the cells grown in the presence of $2 \mu \mathrm{g} / \mathrm{ml} \mathrm{BE}$ was higher than that in untreated cells but lower than that in the cells grown in the presence of $0.5 \mu \mathrm{g} / \mathrm{ml} \mathrm{AmB}$. Combination of $\mathrm{AmB}$ and $\mathrm{BE}$ resulted in a dramatically higher ROS production. After $4 \mathrm{~h}$ of treatment, the ROS level in the cells treated with the combination of $\mathrm{AmB}$ and $\mathrm{BE}$ was nearly three times higher than that in the cells treated with AmB alone.

BE Enhances the Caspase Activity and CaMCA1 Expression in $C$. albicans Induced by AmB Since $C$. albicans caspase and its encoding gene CaMCA1 has been found to play an important role in apoptosis, ${ }^{18)}$ here we investigated the caspase activity by staining the cells with $\mathrm{D}_{2} \mathrm{R}$. As shown in Fig. 4A, the caspase activity in AmB-treated groups were significantly higher than that in the control groups, while there was no significant difference in the caspase activity between the BE-treated groups and the control groups. The combination of $\mathrm{AmB}$ and $\mathrm{BE}$ resulted in an even higher caspase activity, almost three times higher than the groups treated by AmB alone.

Furthermore, the transcription of CaMCA1, which is responsible for caspase activity in C. albicans, was investigated by real time RT-PCR. As shown in Fig. 4B, the transcription of CaMCA1 mRNA in AmB-treated groups was increased.
A
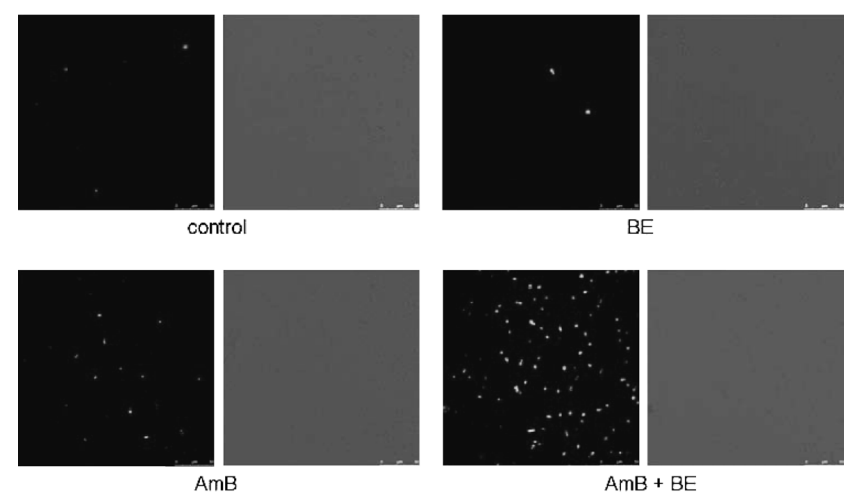

B

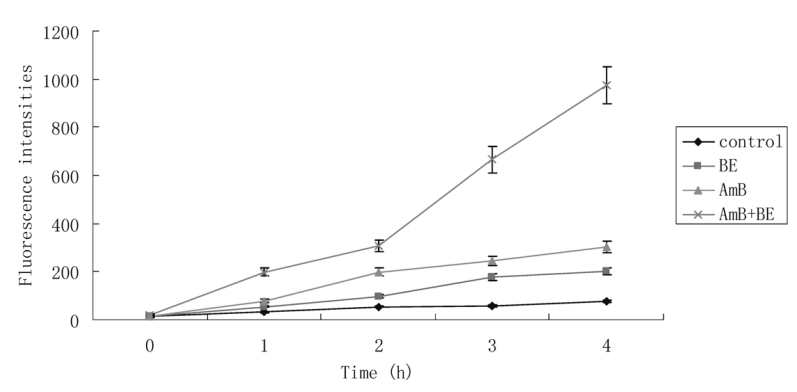

Fig. 3. Effect of $\mathrm{AmB}$ and $\mathrm{BE}$ on ROS Production

C. albicans 0610215 cells were exposed to $0.5 \mu \mathrm{g} / \mathrm{ml} \mathrm{AmB}, 2 \mu \mathrm{g} / \mathrm{ml} \mathrm{BE}$ or a combination. (A) After exposure for $4 \mathrm{~h}$, the intracellular ROS were observed with a Leica TCS $s p^{2}$ confocal scanning laser microscope with excitation at $485 \mathrm{~nm}$ and emission at $520 \mathrm{~nm}$. (B) At the indicated times, aliquots of cells were taken to measure the intracellular ROS by POLARstar Galaxy with excitation at $485 \mathrm{~nm}$ and emission at $520 \mathrm{~nm}$. These data were mean values \pm S.D. from three independent experiments.

Treatment of BE had no significant effect on CaMCA1 expression. When the cells were treated with the combination of $\mathrm{AmB}$ and $\mathrm{BE}$ for $2 \mathrm{~h}$, the transcript level of CaMCA1 mRNA was about five fold higher than the untreated cells.

Deletion of CaMCA1 Attenuates Apoptosis and Caspase Activity in $C$. albicans Induced by AmB Because of the high-level expression of CaMCA1 mRNA in real-time RT-PCR, we investigated the role of CaMCA1 in the death of C. albicans using the CaMCA1 null mutant (Camcal/ Camcal), CAMCA1 reintroduced (CaMCA1-EXP) and wildtype strains $(C A F 2-1){ }^{30)}$ As shown in Fig. 5A, deletion of CaMCA1 could clearly attenuate the cell death induced by AmB. The apoptotic rates were $18 \%, 6 \%$, and $21 \%$ in $C A F 2$ 1, Camcal/Camca1, and CaMCA1-EXP cells, respectively, after being exposed to $0.5 \mu \mathrm{g} / \mathrm{ml} \mathrm{AmB}$ for $4 \mathrm{~h}$. Deletion of CaMCA1 had no significant effect on the cell death induced 
A

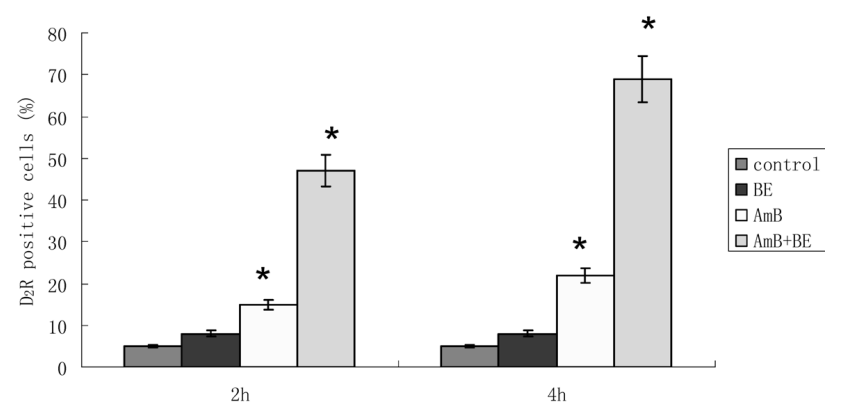

B

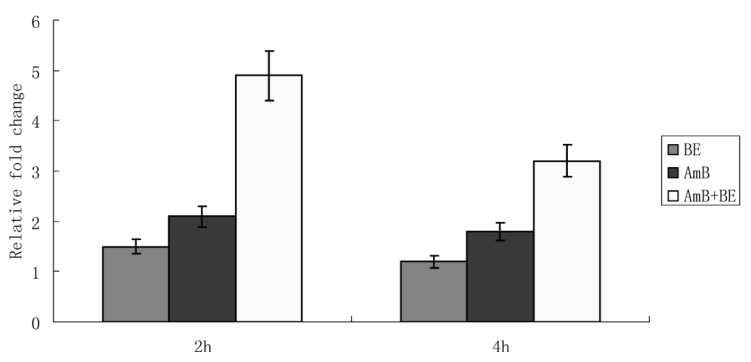

Fig. 4. Effect of $\mathrm{AmB}$ and $\mathrm{BE}$ on Caspase Activity

C. albicans 0610215 cells were exposed to $0.5 \mu \mathrm{g} / \mathrm{ml} \mathrm{AmB}, 2 \mu \mathrm{g} / \mathrm{ml} \mathrm{BE}$ or a combination for 2 or $4 \mathrm{~h}$. (A) The caspase activity was determined by staining the cells with $\mathrm{D}_{2} \mathrm{R}$. (B) Transcription levels of CaMCAl were determined by real-time RT-PCR. The mRNA levels were normalized on the basis of their $A C T 1$ levels. Gene expression was indicated as the fold increase of compounds-treated cells relative to that of the untreated cells. These data were mean values \pm S.D. from three independent experiments

* Indicated $p<0.01$ compared with values from the control groups.

\section{A}

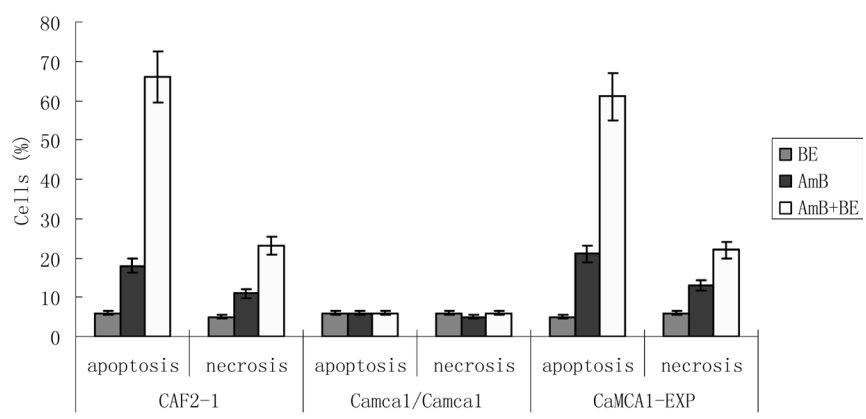

B

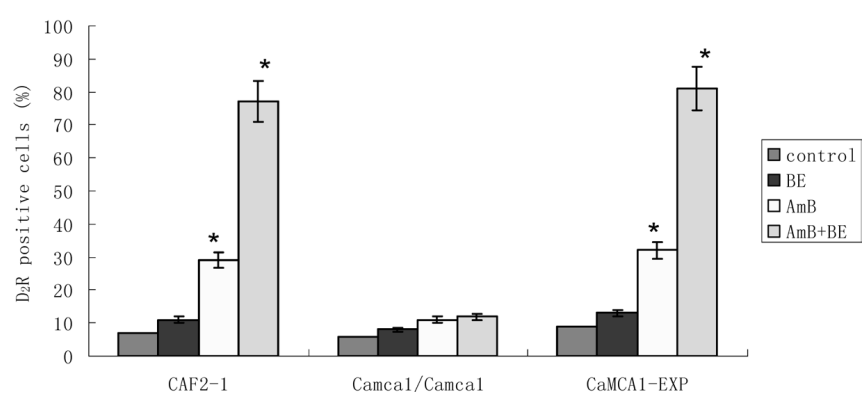

Fig. 5. Role of CaMCA1 in AmB and BE-Induced Cell Death

The wild-type (CAF2-1), CaMCAI null mutant (Camcal/Camcal) and CaMCA1 reintroduced (CaMCA1-EXP) cells were exposed to $0.5 \mu \mathrm{g} / \mathrm{ml} \mathrm{AmB}, 2 \mu \mathrm{g} / \mathrm{ml} \mathrm{BE}$ or a combination for $4 \mathrm{~h}$. (A) Propidium iodide (PI) and FITC-labeled annexin-V were used to determine the rate of cell death, including apoptotic (a) annexin (+) PI $(-)$, and necrotic (n) annexin (+) PI (+) cells. (B) Caspase activity was determined by staining the cells with $D_{2} R$. These data were mean values \pm S.D. from three independent experiments. * Indicated $p<0.01$ compared with values from the control groups. by $2 \mu \mathrm{g} / \mathrm{ml} \mathrm{BE}$ alone. When BE and AmB were used in combination, the apoptotic rate of CAF2-1 were clearly increased (66\%), while the apoptotic rate of Camcal/Camcal almost remains (7\%). Consistent with this, neither AmB alone nor it combined with $\mathrm{BE}$ could induce a clear increase of caspase activity in CaMCA1 null mutant (Fig. 5B). These results indicated that AmB-induced apoptosis in $C$. albicans and the synergy effect of $\mathrm{BE}$ with $\mathrm{AmB}$ was dependent of CaMCA1mediated caspase pathway.

\section{DISCUSSION}

Although AmB is the gold standard of antifungal treatment for the most severe invasive mycoses, adverse effects are common, with nephrotoxicity being the most serious, occurring early in the course of treatment. The severe side effect restricts $\mathrm{AmB}$ clinical application and this has increased the interest in using drug combinations to enhance its efficacies, reduce the dose of $\mathrm{AmB}$ required for therapy to lessen the severity of the side effects. In this work, we demonstrated that $\mathrm{BE}$ could enhance the fungicidal activity of $\mathrm{AmB}$ against $C$. albicans. The checkerboard microdilution assay was used to examine the relationship between $\mathrm{AmB}$ and $\mathrm{BE}$ in terms of their growth inhibitory activities against C. albicans. All the clinical isolates were susceptive to $\mathrm{AmB}$, and $\mathrm{BE}$ also had certain inhibitory activity to the growth of C. albicans although the MIC was relatively high. The FIC index indicated that there was a significantly synergetic interaction between $\mathrm{AmB}$ and BE.

As one of the main ingredients of Chinese herb, Scutellaria baicaleinsis, BE has been studied extensively and reported to show a wide range of biological activities. The inhibitory activity of $\mathrm{BE}$ alone on microorganism was reported several years ago. Fujita et al. showed the synergism between $\mathrm{BE}$ and tetracycline, and $\mathrm{BE}$ and $\beta$-lactams against methicillin-resistant Staphylococcus aureus. Chang et al. reported the synergism of BE and gentamicin against vancomycin-resistant Enterococcus. ${ }^{32,33)}$ We previously found that BE could induce $C$. albicans apoptosis and prevent the formation of C. albicans biofilm. Combination of fluconazole and $\mathrm{BE}$ showed strong antifungal activity against fluconazole-resistant C. albicans isolates. ${ }^{18,31,34)}$ Here our findings of the chemosensitising activity of $\mathrm{BE}$ to AmB suggested the promising application of this compound to the clinical drug-resistant fungi.

Since the finding of apoposis in fungi, induction of cell apoptosis has been considered as an effective and promising way for antifungal therapy. In fact, various antifungal agents, such as plagiochin $\mathrm{E}$ and melittin have already been demonstrated to act as yeast-specific apoptotic inducers. ${ }^{35,36)}$ It is commonly known that AmB can complex with the sterols in the fungal cell membrane and induces structural changes that allow small ions to follow across the cell membrane resulting in cell death. Phillips et al. ${ }^{12)}$ reported that AmB could induce apoptosis of $C$. albicans, indicating a new antifungal mechanism of AmB. Here this mechanism was confirmed in our experiments. Moreover, consistent with the synergistic effect obtained from checkerboard microdilution assay, our cell death detection showed that BE could strikingly enhance AmB-induced C. albicans apoptosis. The apoptosis rate of the cells treated with the combination of $\mathrm{BE}$ and $\mathrm{AmB}$ was 
much higher than that of the cells treated with $\mathrm{BE}$ or $\mathrm{AmB}$ alone. This result was further confirmed by the dramatically increased production of ROS, one of the indicators of cell apoptosis. To our knowledge, this is the first time for reporting that apoptosis is involved in the synergistic effect between AmB and other compounds.

Unlike the extensive study of the mechanisms responsive for mammalian cells apoptosis, the pathways involved in $C$. albicans apoptosis has not been fully revealed. Ras-cAMPprotein kinase A (PKA) was reported to mediate the acetic acid and oxidative stress-induced apoptosis in $C$. albicans. Mutations that blocked Ras-cAMP-PKA signaling (rasl $\Delta$,

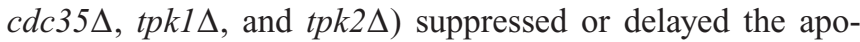
ptotic response, whereas mutations that stimulated signaling (RAS1 ${ }^{\text {vall3 }}$ and pde2 $\Delta$ ) accelerated the rate of entry into apoptosis. ${ }^{13)}$ We previously found that CaMCA1, a homologue of $S$. cerevisiae metacaspase $Y C A 1$, was involved in $\mathrm{H}_{2} \mathrm{O}_{2}$-induced apoptosis in C. albicans. ${ }^{30)}$ However, the basis of AmB-induced C. albicans apoptosis is still unclear. Here we found that the caspase activity was significantly increased when the cells were treated with AmB alone, combination of $\mathrm{BE}$ and $\mathrm{AmB}$ resulted in an even higher caspase activity. Moreover, real time RT-PCR experiment showed that the mRNA level of CaMCA1, the only gene reported to be responsible for the caspase activity in $C$. albicans, was increased upon the treatments of AmB alone or combined with $\mathrm{BE}$. To further confirm the involvement of CaMCA1 in AmB-induced apoptosis and the synergy effect, we tested the cell death of CaMCA1 null mutant. Our experiments showed that deletion of CaMCA1 could clearly attenuate the cell death and caspase activity induced by AmB. These results indicated that CaMCA1-mediated caspase pathway was involved in AmB-induced apoptosis. Of note, the apoptosis of C. albicans induced by BE seemed independent of CaMCA1mediated caspase pathway because there were no significant changes of caspase activity or CaMCAl expression in the cells treated with $\mathrm{BE}$ alone. Also CaMCA1 mutant showed similar death rate with the wild-type strain in the presence of BE. However, when the cells were treated with the combination of $\mathrm{BE}$ and $\mathrm{AmB}$, the CaMCA1-mediated caspase pathway was hyperactivated, indicating the involvement of CaMCA1-mediated caspase pathway in AmB-induced apoptosis and the synergistic action of $\mathrm{BE}$ and $\mathrm{AmB}$.

Acknowledgements This work was supported by the National Natural Science Foundation of China (No. 30870105). We thank Professor Theodore C. White of Seattle Biomedical Research Institute (Seattle, Washington) and Jun Gu of Changhai Hospital (Shanghai, China) for kindly providing the isolates used in this study.

\section{REFERENCES}

1) Fidel P. L. Jr., Adv. Dent. Res., 19, 80-84 (2006).

2) Perlroth J., Choi B., Spellbergn B., Med. Mycol., 45, 321-346 (2007).

3) Fanos V., Cataldi L., J. Chemother, 12, 463-470 (2000).
4) Matsuoka S., Murata M., Biochim. Biophys. Acta, 1564, 429-434 (2002).

5) Mayer J., Doubek M., Doubek J., Horký D., Scheer P., Stepánek M., J. Infect. Dis., 186, 379-388 (2002).

6) Sugar A. M., Hitchcock C. A., Troke P. F., Picard M., Antimicrob. Agents Chemother, 39, 598-601 (1995).

7) Lewis R. E., Lund B. C., Klepser M. E., Ernst E. J., Pfaller M. A., Antimicrob. Agents Chemother, 42, 1382-1386 (1998).

8) Cowen L. E., Anderson J. B., Kohn L. M., Annu. Rev. Microbiol., 56, $139-165$ (2002).

9) Madeo F., Frohlich E., Frohlich K. U., J. Cell Biol., 139, 729-734 (1997).

10) Madeo F., Fröhlich E., Ligr M., Grey M., Sigrist S. J., Wolf D. H., J. Cell Biol., 145, 757-767 (1999).

11) Silva R. D., Sotoca R., Johansson B., Ludovico P., Sansonetty F., Mol. Microbiol., 58, 824-834 (2005).

12) Phillips A. J., Sudbery I., Ramsdale M., Proc. Natl. Acad. Sci. U.S.A., 100, 14327-14332 (2003).

13) Phillips A. J., Crowe J. D., Ramsdale M., Proc. Natl. Acad. Sci. U.S.A., 103, 726-731 (2006).

14) Madeo F., Herker E., Maldener C., Wissing S., Lachel S., Mol. Cell, 9, $911-917$ (2002).

15) Madeo F., Herker E., Wissing S., Jungwirth H., Eisenberg T., Curr. Opin. Microbiol., 7, 655-660 (2004).

16) Wadskog I., Maldener C., Proksch A., Madeo F., Adler L., Mol. Biol. Cell, 15, 1436-1444 (2004).

17) Khan M. A., Chock P. B., Stadtman E. R., Proc. Natl. Acad. Sci. U.S.A., 102, 17326-17331 (2005).

18) Cao Y. Y., Dai B. D., Wang Y., Huang S., Xu Y. G., Cao Y. B., Gao P. H., Zhu Z. Y., Jiang Y. Y., Int. J. Antimicrob. Agents, 32, 73-77 (2008).

19) Ishige K., Schubert D., Sagara Y., Free Radic. Biol. Med., 30, 433446 (2001).

20) Van Leyen K., Kim H. Y., Lee S. R., Stroke, 37, 3014-3018 (2006).

21) Chang P. C., Li H. Y., Tang H. J., Liu J. W., Wang J. J., Chuang Y. C., J. Microbiol. Immunol. Infect., 40, 56-61 (2007).

22) Lapchak P. A., Maher P., Schubert D., Neuroscience, 150, 585-591 (2007).

23) Lin H. Y., Shen S. C., Lin C. W., Biochim. Biophys. Acta, 1773, 1073-1086 (2007).

24) Marr K. A., White T. C., van Burik J. A. H., Clin. Infect. Dis., 25, 908-910 (1997).

25) Marr K. A., Lyons C. N., Rustad T. R., Antimicrob. Agents Chemother. 42, 2584-2589 (1998).

26) Marr K. A., Lyons C. N., Ha K., Antimicrob. Agents Chemother, 45, $52-59$ (2001).

27) National Committee for Clinical and Laboratory Standards, Reference Method for Broth Dilution Antifungal Susceptibility Testing of Yeasts: Approved Standard M27-A. NCCLS, Wayne, PA, U.S.A., 1997.

28) Odds F. C., J. Antimicrob. Chemother, 52, 1-9 (2003)

29) Wang Y., Cao Y. Y., Jia X. M., Cao Y. B., Gao P. H., Jiang Y. Y., Free Radic. Biol. Med., 40, 1201-1209 (2006).

30) Cao Y. Y., Huang S., Dai B. D., Zhu Z. Y., Lu H., Dong L. L., Cao Y. B., Wang Y., Gao P. H., Chai Y. F., Jiang Y. Y., Fungal Genet. Biol., 46, 183-189 (2009).

31) Dai B. D., Cao Y. Y., Huang S., Xu Y. G., Gao P. H., Wang Y., Jiang Y. Y., J. Microbiol. Biotechnol., 19, 803-809 (2009).

32) Chang W. T., Shao Z. H., Yin J. J., Eur. J. Pharmacol., 566, 58-66 (2007).

33) Fujita M., Shiota S., Kuroda T., Hatanok T., Yoshida T., Mizushima T., Tsuchiya, T., Microbiol. Immunol., 49, 391-396 (2005).

34) Huang S., Cao Y. Y., Dai B. D., Sun X. R., Zhu Z. Y., Cao Y. B., Wang Y., Gao P. H., Jiang Y. Y., Biol. Pharm. Bull., 31, 2234-2236 (2008).

35) Park C., Lee D. G., Biochem. Biophys. Res. Commun., 394, 170-172 (2010).

36) Wu X. Z., Chang W. Q., Cheng A. X., Sun L. M., Lou H. X., Biochim Biophys. Acta, 1800, 439-447 (2010). 